\title{
Tsafon
}

Revue d'études juives du Nord

73 | 2017

Connaissance de la Shoah : témoignages, enseignements, visites

\section{Enseigner la Shoah à l'école primaire}

\section{Monique Heddebaut}

\section{(2) OpenEdition \\ Journals}

Édition électronique

URL : https://journals.openedition.org/tsafon/283

DOI : $10.4000 /$ tsafon.283

ISSN : 2609-6420

Éditeur

Association Jean-Marie Delmaire

Édition imprimée

Date de publication : 1 juin 2017

Pagination : 47-58

ISSN : $1149-6630$

\section{Référence électronique}

Monique Heddebaut, «Enseigner la Shoah à l'école primaire », Tsafon [En ligne], 73 | 2017, mis en ligne le 31 mai 2018, consulté le 24 juin 2021. URL : http://journals.openedition.org/tsafon/283 ; DOI : https://doi.org/10.4000/tsafon.283 


\title{
Enseigner la Shoah à l'école primaire
}

\author{
Monique Heddebaut ${ }^{*}$
}

Au cours des années 1970-1980, l'enseignement de la Shoah était survolé, voire ignoré. Or le silence n'est plus de mise aujourd'hui, il est même pire que tout. Dans l'enseignement de l'histoire, même à l'école élémentaire, il est devenu un moment fort, jamais anodin, mais qui ne peut être abordé comme n'importe quel autre événement particulier de l'histoire contemporaine par rapport aux autres chapitres étudiés durant l'année. Sa dimension tragique, l'énormité des chiffres dépassent l'entendement. Tout concourt à lui donner une charge émotionnelle qui pourrait submerger les élèves, mais également les enseignants.

Faut-il alors intégrer l'histoire de la Shoah, dans un temps limité, dans une continuité logique et chronologique sans en faire un moment particulier du programme ? Comment la percevoir, comment toucher la sensibilité des élèves sans pour autant choquer ? Comment faire face à des ricanements, des remarques antisémites ou racistes ou à d'autres « comportements déplacés »?

Telles sont les nombreuses questions que se posent les enseignants qui se risquent à aborder ce pan de notre histoire, inscrit dans le programme, avec un jeune public.

\section{L'histoire de la Shoah à l'école primaire}

Après le discours du président de la République Jacques Chirac (1995-2007) qui reconnaissait le 16 juillet 1995 la responsabilité de l'État français dans la déportation des juifs et après les conclusions de la

\footnotetext{
* Enseignante à l'école René Cassin de Flines-lez-Râches. Membre de la Commission historique du Nord et de l'association du musée de la Résistance de Bondues.
} 
Mission Mattéoli sur les spoliations en $1997^{1}$, le ministère de l'Éducation nationale par la voix de Jacques Lang avait pris la mesure des reconnaissances officielles et institutionnelles en préconisant, en 2000, que la Seconde Guerre soit étudiée de façon plus approfondie :

\begin{abstract}
Je suis très attaché au rôle dévolu à l'Éducation nationale dans la transmission et la préservation de la mémoire. Aussi, je souhaite très vivement que, dans les classes, les professeurs et les élèves évoquent ces pages sombres de notre histoire et réfléchissent à leur signification profonde à un moment où l'Europe est à nouveau le théâtre de conflits qui ne sont pas malheureusement, sous certains aspects, sans rappeler ceux qui seront remémorés. ${ }^{2}$
\end{abstract}

Quelques années plus tard, le 13 février 2008, le président Nicolas Sarkozy (2007-2012) annonçait lors du dîner annuel du CRIF une nouvelle initiative mémorielle concernant les élèves de CM2. Il demandait que

...chaque année, à partir de la rentrée scolaire 2008, tous les enfants de CM2 se voient confier la mémoire d'un des 11000 enfants français victimes de la Shoah [...]. Peut-on trouver plus noble dessein que de permettre à ces enfants martyrs d'être les pédagogues éclairés de nos propres enfants ?

L'initiative suscita immédiatement de vives réactions, hostiles ou pour le moins réservées, des mises en garde venant de l'Association des Professeurs d'Histoire-Géographie (APHG), du Comité de Vigilance face aux Usages de l'Histoire (CVUH), des historiens Annette Wieviorka et Henri Rousso, de Boris Cyrulnik et de Simone Veil pour ne citer que les principaux.

Il est inimaginable, insoutenable, dramatique et, surtout, injuste d'infliger cela à des petits de 10 ans ! On ne peut pas demander à un enfant de s'identifier à un

\footnotetext{
${ }^{1}$ Par lettre du 5 février 1997, M. Alain Juppé, alors Premier ministre, avait demandé à M. Jean Mattéoli, ancien déporté de la Résistance et président du Conseil économique et social, d'étudier les conditions dans lesquelles les biens appartenant aux personnes considérées comme juives par l'occupant ou les autorités de Vichy avaient été confisqués, ou d'une manière générale, acquis par fraude, violence ou vol dans le cadre de la politique antisémite qui a sévi en France entre 1940 et 1944. Mission d'étude sur la spoliation des Juifs de France - rapport au Premier ministre, dite « Mission Mattéoli » 118 p., déc. 1997.

2 BO du 20/04/2000, Journée nationale du souvenir de la Déportation et du $55^{\mathrm{e}}$ anniversaire de la Victoire du 8 mai 1945.

${ }^{3}$ http://www.cndp.fr/crdp-reims/memoire/enseigner/memoire_vichy/14shoah_cm $2 . h t m$. Voir le dossier complet des réactions.
} 
enfant mort. Cette mémoire est beaucoup trop lourde à porter. Nous-mêmes, anciens déportés, avons eu beaucoup de difficultés, après la guerre, à parler de ce que nous avions vécu, même avec nos proches. ${ }^{4}$

En revanche, le grand rabbin Joseph Sitruk se déclarait «parfaitement en accord avec l'idée de Nicolas Sarkozy»et Serge Klarsfeld considérait cette initiative comme « extraordinaire » et dans la continuité du travail de mémoire qui avait abouti en 1978 et qui lui avait permis d'établir les noms des 76000 victimes de la Shoah en France.

Très rapidement Hélène Waysbord-Loing, suite à la lettre de mission du 27 février 2008 du président Sarkozy, présenta un rapport qui était l'aboutissement d'une réflexion collective et qui permettait de poser le cadre, d'aider les enseignants, mais également de calmer les réticences ${ }^{5}$. L'enseignement de la Shoah qui figurait dans les programmes de l'école élémentaire depuis 2002 était réaffirmé dans ceux de 2008 et accompagné de supports pédagogiques très documentés ${ }^{6}$. Plusieurs raisons à cette relance: les témoins et les victimes disparaissaient progressivement et les enseignants avaient désormais à faire à des élèves nés après la chute du Mur de Berlin dans un contexte européen dont l'épine dorsale était l'amitié franco-allemande après des décennies d'hostilité et de conflits. À noter de surcroit l'introduction de l'extermination des Tsiganes dans les programmes, mais sans précisions aucunes, et dans le cas présent, sans outils documentaires ou pédagogiques spécifiques pour les enseignants ${ }^{7}$.

L'approche faisait donc débat. L'un des points sensibles était l'une des thématiques retenues : «L'histoire des enfants morts, mais aussi des histoires individuelles et collectives de vies et de survies ${ }^{8}$. Il était rappelé que le travail mené en classe sur les victimes étudiées par le biais de récits et de figures particulières, devait être entrepris avec le souci de ne pas traumatiser un public jeune, mais avec la volonté de leur donner

\footnotetext{
${ }^{4}$ Interview de Simone Veil, 15 février 2008, L'Express, numéro 2956.

5 Rapport remis à Xavier Darcos, ministre de l'Éducation nationale, par Hélène Waysbord-Loing, inspectrice générale honoraire de l'Éducation nationale, présidente de l'association de la Maison d'Izieu, $8 \mathrm{p}$. http://media.education.gouv.fr/file/06_juin/77/7/Rapport_Shoah_Waysbord_28777.pdf ${ }^{6}$ Enseignement de la Shoah à l'école élémentaire (BO n⿳亠丷厂 $29 \mathrm{du}$ 17/07/2008) et Mémoire et histoire de la Shoah à l'école, Ressources pour faire la classe, CNDP, 2008, 39 p.

7 «L'extermination des Juifs et des Tziganes par les nazis: un crime contre l'humanité ». Or, les Tsiganes étaient assignés à résidence ou internés en France. Les seuls cas de déportations collectives concernent le Nord/Pas-de-Calais qui était rattaché au Haut Commandement Militaire de Bruxelles.

${ }^{8}$ Enseignement de la Shoah à l'école élémentaire ... p. 27.
} 
une leçon de vie et d'énergie, et qu'il pouvait être élargi aux enfants cachés et sauvés. Aux enseignants de trouver une signification universelle à partir de cas particuliers, de passer du singulier au général, puis à l'universel. Parmi les pistes proposées dans le rapport, les enseignants pouvaient retrouver les traces d'enfants d'une école ou d'un quartier, d'un contexte historique en fonction du contexte de la classe, des ressources de proximité, des interrogations des élèves.

\section{Expérience personnelle}

Dans ma pratique de l'enseignement de l'histoire en tant que professeur des écoles, j'ai toujours cherché à m'appuyer sur des témoignages, sur des documents d'archives issus des fonds locaux, départementaux ou régionaux. Afin de rendre la Seconde Guerre mondiale plus concrète, plus palpable auprès de mes élèves, j'avais entrepris en 2004 des recherches personnelles sur cette période dans les archives de Flines-lez-Râches, la commune où j'avais été nommée. Et c'est là que je découvris plusieurs documents qui me laissèrent perplexe. En effet, le 24 septembre 1940 le capitaine et commandant Schnatz réclamait aux maires de l'arrondissement de Douai le nom de tous les juifs évacués et/ou revenus et la liste de tous les magasins juifs, exigeant une réponse pour le 27 courant par l'intermédiaire du sous-préfet ${ }^{9}$. Dans un autre document en date du $1^{\mathrm{er}}$ août 1941 , le maire de Flines l'informait « qu'il existait précédemment dans [sa] commune trois hommes reconnus comme Juifs, mais que ces trois personnes » avaient quitté la commune le 19 mai 1940 et qu'il existait dans sa commune « une entreprise appartenant aux personnes sus-visées (manufacture de confections) ${ }^{10}$. Il est exact que Gaston Michel avait créé à Flines un atelier de confection en 1927 et que sa famille avait quitté le Nord dans le mouvement général de fuite et de panique suite à l'invasion allemande en mai 1940. Comme le révélaient les archives, ils s'étaient réfugiés en zone libre à Caussade (Tarn-en-Garonne) $^{11}$. Mais j'ignorais alors complètement, jusqu'à ce jour précis, qu'un épisode de la Shoah s'était déroulé à Flines et, qui plus est, au bout de la rue où j'habitais. De plus, la tradition orale, l'association des anciens combattants ou celle d'histoire locale de la commune, étaient

\footnotetext{
${ }^{9}$ Archives municipales de Flines-lez-Râches, non cotées.

${ }^{10}$ Idem.

${ }^{11}$ Monique Heddebaut, «De la difficulté d'être français en 1940-1941 à Flines-lesRâches et ailleurs... », Pays de Pévèle, n 55, 2004, pp. 35-42.
} 
restées silencieuses sur ces faits. Rien n'avait été publié à ce sujet. Avait alors commencé une recherche inquiète, une quête afin de connaître l'itinéraire de cette famille et surtout leur devenir dans la tourmente de la Seconde Guerre mondiale.

Ce fut le déclencheur d'une recherche qui se transforma en recherches croisées puisqu'à la même époque la petite-fille de Gaston Michel, Monique Michel-Biezunski, qui habitait désormais en région parisienne, travaillait sur l'histoire de sa propre famille et sur les spoliations dont elle avait été victime ${ }^{12}$. Je n'étais pas au bout de mes surprises. Après avoir fait sa connaissance, j'apprenais que trois des petits-enfants de Gaston Michel avaient fréquenté, en 1939-1940, l'école de la rue Dupire - aujourd'hui René Cassin - où j'enseignais. Je lui proposais donc tout naturellement de venir parler devant mes élèves de CM1. Monique Biezunski-Michel est venue accompagnée le 18 mai 2007 de sa sœur, Nicole Malamet-Michel, qui réside en Israël, et de son fils. Je conçus donc spontanément le projet de quelques séquences sur le régime de Vichy et la Shoah, autour des persécutions raciales en France, mais à partir d'événements qui s'étaient déroulés dans la commune. L'histoire de la Shoah est trop souvent et essentiellement abordée au travers des camps d'extermination situés à plus de mille kilomètres en Europe de l'est où la responsabilité de l'État français et du régime de Vichy est occultée ou si peu développée. C'est dans ce contexte, mais avec un peu d'avance sur les recommandations de l'institution, que je décidais de mener ces quelques séquences dans ma classe, avec le CM1 que j'avais en charge cette année-là, mais aussi plus largement dans le cycle 3 (CE2CM1 et CM2) où j'enseignais l'histoire dans le cadre d'un échange de services entre collègues. Le milieu ne posait a priori pas de difficultés particulières : Flines-lez-Râches est une commune de 5000 habitants, de moyenne importance, dans le Douaisis très urbanisé et industriel, à la périphérie de la Pévèle rurale.

Avant d'aborder précisément la Shoah et l'antisémitisme grâce aux témoignages de ces deux anciennes élèves dont les familles avaient échappé aux persécutions raciales extrêmes, je m'étais demandé quelles pouvaient être les représentations d'enfants de CM1, dans la tranche d'âge 8/9 ans. Je leur avais donc distribué un questionnaire succinct auquel ils devaient répondre anonymement. Je craignais d'avoir des réponses stéréotypées où chacun reprenait les propos du dernier qui avait

\footnotetext{
${ }^{12}$ Monique Heddebaut, Les persécutions raciales dans l'arrondissement de Douai Juifs et Tsiganes, Tsafon, hors-série nº 4, Villeneuve d'Ascq, 2008, 142 p.
} 
parlé. Je leur proposais quelques mots-clés tels : camp de concentration, Auschwitz, juif, Seconde Guerre ; sachant qu'en CE2 ils avaient étudié la Seconde Guerre mondiale, mais dans le cadre des commémorations nationales et dans l'optique de la Libération du territoire et de la paix retrouvée. Je souhaitais savoir ce dont ils avaient entendu parler, quelles étaient leurs représentations, s'ils avaient des connaissances particulières et quelles étaient leurs attentes. La majorité connaissait ces termes tout en fournissant des réponses extrêmement vagues, mais manifestait de la curiosité. Dans ma pratique au cycle 3, je m'étais rendu compte que les enfants n'associaient pas le terme «juif» à une religion, mais qu'il recouvrait une catégorie très imprécise, qu'ils n'avaient d'ailleurs que peu de notions sur les religions en général - sur l'islam, encore moins sur le judaïsme et très souvent pas même sur l'histoire du christianisme. Ce qui m'obligeait régulièrement à m'arrêter sur les faits religieux comme élément de compréhension de notre patrimoine et $\mathrm{du}$ monde contemporain alors qu'ils ne faisaient pas à l'époque l'objet d'un enseignement spécifique, et surtout parce qu'ils apparaissent en permanence dans l'actualité. Or, c'est indispensable, ne serait-ce que lorsqu'on étudie l'histoire des Guerres de religion et la naissance du protestantisme en France au programme du cycle 3. Ce n'est que très récemment que l'importance de cet enseignement a été soulignée ${ }^{13}$. Cela permet également de ne pas définir le peuple juif uniquement en référence à la Shoah.

À mon très grand étonnement, à la question : «Qu'est-ce qu'un juif? » un élève de neuf ans avait écrit : "On le reconnaît à son nez ». Cela me conforta dans mon projet de traiter la Shoah, et à partir de la France. Cela devint une nécessité et même une urgence.

J'annonçai ensuite aux élèves que j'avais réussi à retrouver une ancienne élève qui avait fréquenté notre école entre 1939 et mai 1940, que sa famille avait quitté Flines et qu'elle n'était pas revenue en raison des lois décidées par le gouvernement de l'époque contre les juifs. Puis je demandai à chaque élève de proposer une question qu'il poserait à Monique Michel.

\footnotetext{
${ }^{13}$ Rapport annexé à la loi $\mathrm{n}^{\circ} 2005-380$ d'orientation et de programme pour l'avenir de l'école du 23 avril 2005 : «Il convient dans le respect de la liberté de conscience et des principes de laïcité et de neutralité du service public, d'organiser dans l'enseignement public la transmission de connaissances et de références sur le fait religieux et son histoire ».

http://eduscol.education.fr/cid46675/enseignement-laique-des-faits-religieux.html
} 
1 - Quel âge avez-vous?

2 - Combien étiez-vous dans votre famille?

3 - Quel est votre métier ? Est-il dur?

4 - Avez-vous des enfants, des petits-enfants ?

5 - Avez-vous connu la guerre? Laquelle?

6 - Quel âge aviez-vous quand la guerre a commencé ? Quand la guerre s'est terminée?

7 - Votre père a-t-il fait la guerre ?

8 - Les juifs avaient-ils le droit d'être dans l'armée?

9 - Votre maison a-t-elle été bombardée ?

10 - Vous rappelez-vous si les chemins de fer ont été détruits ?

11 - La guerre a duré six ans. Donc, comme le président de la République est élu pour cinq ans, comment avez-vous fait pour voter?

12 - Pourquoi êtes-vous venue dans notre école étant petite ? Où habitiezvous?

13 - Comment s'appelait l'école à cette époque-là ?

14 - Comment sont morts vos parents ? À quel âge ? Ont-ils survécu à la guerre?

15 - Des gens de votre famille sont-ils morts pendant la guerre? Dans un camp d'extermination?

16 - Dans quelle ville habitiez-vous quand les nazis sont arrivés ?

17 - Vous, votre frère, votre sœur étiez-vous toujours ensemble ? À quel âge est mort votre frère?

18 - Comment vous êtes-vous nourrie pendant la guerre ? Comment avez-vous survécu à la guerre ?

19 - Vous rappelez-vous des sentiments que vous aviez pendant cette période?

20 - Avez-vous de mauvais souvenirs de la guerre ? Lesquels ? En avezvous de bons?

21 - Êtes-vous déjà allée dans un camp de concentration ? En Allemagne?

22 - Votre mari est-il allé à la guerre ?

23 - Comment sont les tombes des juifs?

24 - Comment se passe un enterrement juif, un enterrement musulman ?

25 - Que dites-vous des profanations de tombes?

Leur arrivée fit figure d'événement et les enfants furent très impressionnés, d'abord par leur âge, par leur qualité de témoins ayant vécu la guerre et découvrant concrètement ce qu'était «être enfant 
pendant et dans la guerre, avoir être confronté à des arrestations, à la mort ». Après les réponses précises aux questions rédigées au préalable, la discussion fut menée à bâtons rompus : Monique et Nicole racontèrent la guerre, leur guerre, exemples et anecdotes à l'appui.

\section{Quelques réflexions et pistes pédagogiques}

Un tel témoignage était tout à fait exceptionnel et inespéré pour les élèves de l'école René Cassin de Flines. D'anciens élèves et mêmes leurs parents m'en parlent encore. L'on constate que le témoignage des acteurs de l'histoire peut et doit faire appel à l'émotion. Mais si l'enseignement de la Shoah fait appel à l'affectif, il ne peut à lui seul donner la matière d'un cours d'histoire, il ne peut le remplacer. Se contenter de l'émotionnel n'est pas faire de l'histoire. Dans le cas présent le témoignage fut un vecteur, un déclencheur incomparable pour rendre l'histoire plus concrète, plus compréhensible, comme à portée de mains, pour des enfants de cette tranche d'âge qui avaient déjà une première approche de la Seconde Guerre mondiale.

Dans un deuxième temps, la réflexion et la prise de distance furent suscitées. Après ce témoignage pensé comme une introduction à l'étude de la Shoah, d'autres séquences furent consacrées au contexte : l'entrée en guerre avec une attention particulière pour l'étude de la France divisée en zones où il apparaissait clairement que le Nord/Pas-de-Calais dépendait de Bruxelles (ce qui est rarement souligné dans les manuels scolaires), le régime de collaboration, le nazisme et l'antisémitisme, la résistance, tout en ayant conscience de l'ampleur des sujets à traiter dans le cadre des programmes scolaires. Les enseignants sont actuellement confrontés à des choix d'autant qu'en cette période de commémoration du Centenaire de la Grande Guerre, pour les années 2014-2018, les recommandations de l'institution sont assez pressantes et tentantes. $\mathrm{Si}$ l'on considère toutes les commémorations possibles dans le cadre de l'Éducation nationale, force est de constater que les différentes mémoires entrent en concurrence.

Mais la condition sine qua non pour un réel enseignement de la Shoah est la nécessaire connaissance de cette période pour faire face aux questions, aux affirmations et aux incompréhensions des élèves qui pourraient désarçonner plus d'un enseignant. Avec une pratique régulière de l'enseignement de l'histoire de la Shoah, il m'a été possible de concevoir différentes situations pédagogiques, mais au préalable de 
réfléchir aux conditions devant être réunies et mises en place afin d'éviter certains écueils :

- Adopter avant toute chose une pédagogie active centrée sur les élèves : être attentif à leurs préoccupations, leurs représentions, leur vécu et leur contexte familial.

- Définir le terme Shoah, son processus et utiliser une terminologie précise y compris sur les responsabilités des États, des régimes. Ainsi à la lecture du livre d'Anne Frank et de sa biographie, une élève d'une autre classe avait conclu que la petite fille était morte de maladie - du typhus à Bergen-Belsen, mais qu'elle n'avait pas été gazée par les nazis. Dans les camps, la mort pouvait donc être programmée sous des formes diverses, et pas seulement dans les chambres à gaz. D'où l'importance de débusquer l'amalgame qui est parfois constaté dans la tête des élèves entre camp de concentration et d'extermination, de montrer ce qui relève de la mort immédiate, industrielle et de la mort lente, d'identifier précisément les victimes et les raisons de leur arrestation et de leur déportation.

- Privilégier l'accès à des sources historiques primaires en signalant leur origine (victimes ou bourreaux). Si les témoignages rendent l'histoire plus réelle pour les élèves en présentant des itinéraires, des destinées individuelles par les témoignages, récits ou films, il est impératif de les traduire en chiffres.

- Penser à l'impact des documents visuels ou écrits présentés : les photos, tel le cliché emblématique du petit garçon du ghetto de Varsovie qui tient ses mains en l'air face aux soldats armés ou bien celui de rescapés des camps de concentration derrière des fils barbelés ou encore celui des cadavres ambulants en pyjamas rayés, peuvent choquer des enfants de CM1-CM2. Les journaux qui sont destinés à un jeune public et auxquels sont abonnées beaucoup d'écoles utilisent plus volontiers des dessins pour illustrer la Shoah.

Beaucoup de réticences s'étaient exprimées sur la nécessité, sur la façon d'enseigner la Shoah à l'école élémentaire. Il est vrai que notre histoire est constituée, émaillée de conflits et d'épisodes douloureux étudiés au travers de témoignages poignants, mais parfois d'une très grande dureté sans que personne ne s'en émeuve. Pour qui étudie en CM2 les Guerres de religion, il est impossible de ne pas utiliser l'iconographie sur le massacre de la Saint-Barthélemy. Celui qui utilise «Le feu» d'Henri Barbusse ou les «Lettres de poilus» ne peut ignorer ou minorer les conditions de vie des soldats de la Grande Guerre. Mais 
on nous rétorquera que la Saint-Barthélemy s'est déroulée voici plus de cinq cents ans et qu'en 1914-1918 les morts et les disparus étaient des hommes - devenus des héros - pas des enfants. Le temps est passé, d'où une prise de distance de fait.

- Il convient d'éviter les simulations susceptibles de pousser les élèves à s'identifier avec les bourreaux ou les victimes. Et se contenter de traiter les bourreaux de «monstres inhumains» n'apporte pas d'explication suffisante. Tout comme confier à un enfant de CM2 la mémoire d'un enfant particulier gazé à Auschwitz avait immédiatement été écarté. Mais il est possible d'étudier des différentes réactions des victimes, les nombreuses formes de résistance au nazisme en France, en Allemagne... Les réseaux d'entraide et l'histoire des Justes parmi les nations trouvent ici toute leur place. Parmi les différentes ressources documentaires Le grenier de Sarah, qui est un site du Mémorial de la Shoah, aborde avec une approche originale et dans le respect de la sensibilité des enfants de 8-12 ans la vie quotidienne des enfants juifs de France pendant la Seconde Guerre mondiale, les différents visages de la persécution des juifs en France ${ }^{14}$.

- Veiller à replacer les événements dans leur contexte historique et dans une perspective tout à la fois large et nuancée, mais également montrer que la Shoah n'était pas inévitable, avec toutes les leçons à en tirer. Et si l'on compare les souffrances d'un groupe à celles d'un autre, ne pas les relativiser ni mettre les mémoires en concurrence.

- Encourager les élèves à approfondir les aspects de l'histoire et de la mémoire aux niveaux local, régional, national et mondial et les inviter à participer et à réfléchir aux cérémonies de commémoration. Cela permet de mettre l'accent sur la citoyenneté. Un de mes élèves avait lu devant le monument aux morts de Flines, lors de la commémoration du 8 mai 1945, un extrait du "Journal d'Anne Frank» où celle-ci attendait avec impatience la rentrée de septembre pour repartir enfin en classe.

- L'approche interdisciplinaire (éducation civique et morale, littérature, art, histoire des arts) telle qu'elle figure désormais dans les programmes permet plusieurs entrées, aide à mieux comprendre la Shoah et donne un ancrage plus solide. Si être juif est souvent - trop souvent défini uniquement en référence à la Shoah et non par rapport au judaïsme, mettre l'accent sur la culture yiddish permettrait d'entrevoir la culture quasi effacée par la Seconde Guerre mondiale et le nazisme.

\footnotetext{
${ }^{14} \mathrm{http}: / /$ www.grenierdesarah.org/index.php/fr/
} 
Panneau de bienvenue à Monique et Nicole Michel conçu par les élèves de CM1 de l'école René Cassin à Flines-lez-Râches, 2007

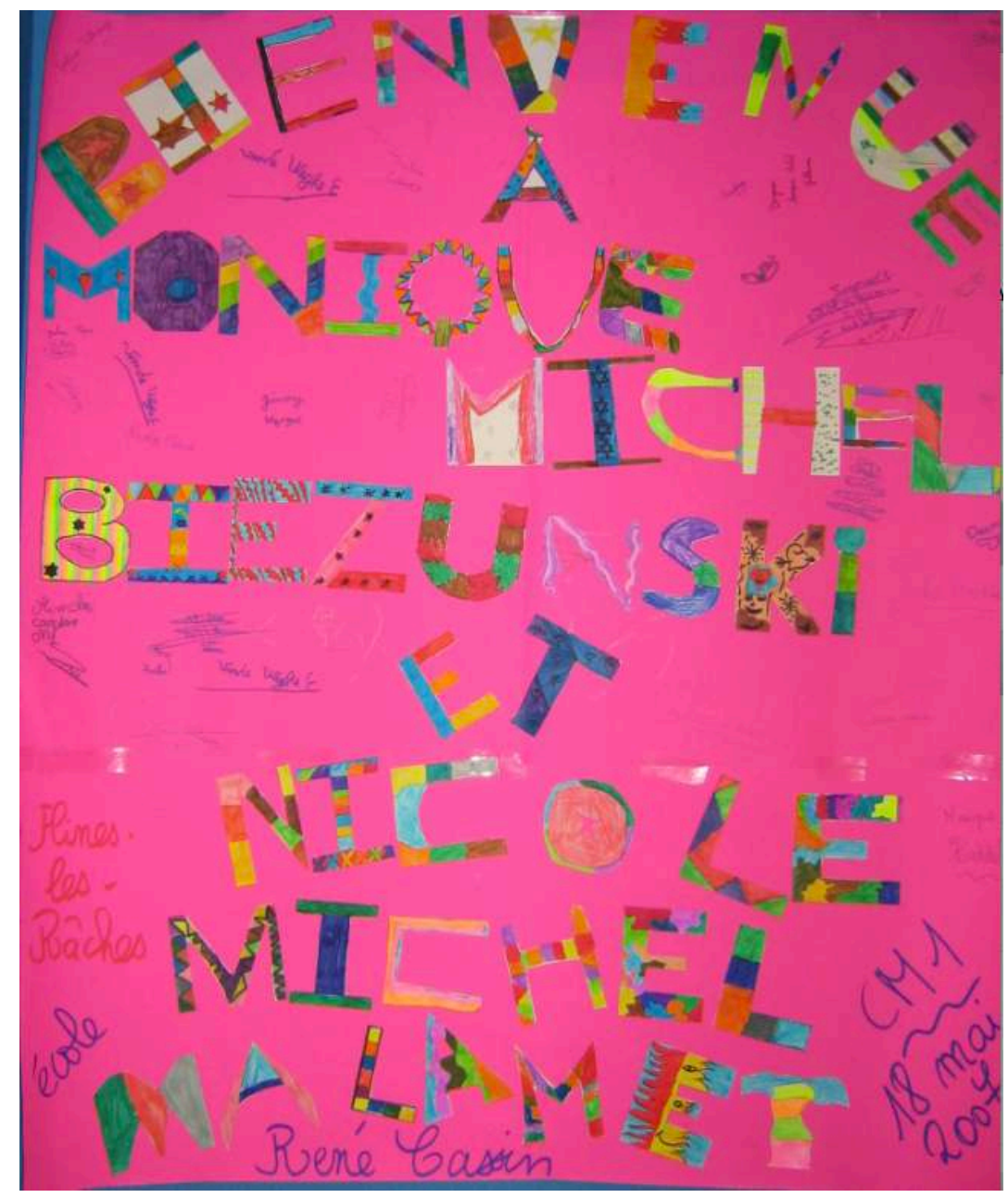

Photo de Monique Heddebaut 
Nicole et Monique Michel face aux élèves de CM1 de l'école René Cassin à Flines-lez-Râches, 2007

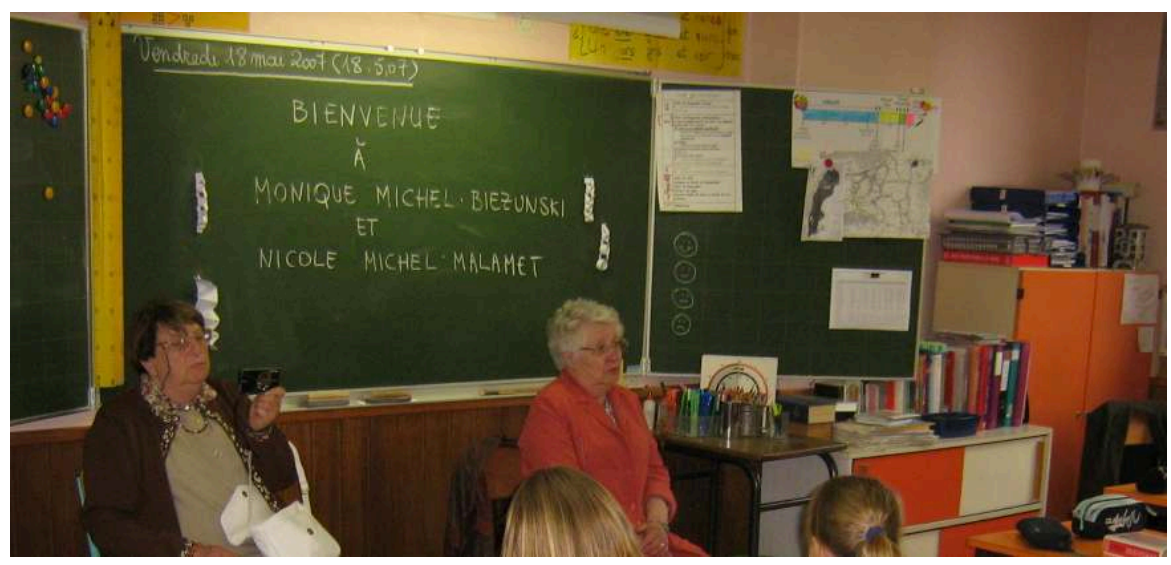

Nicole et Monique Michel dans la cour de récréation de l'école René Cassin à Flines-lez-Râches, 2007

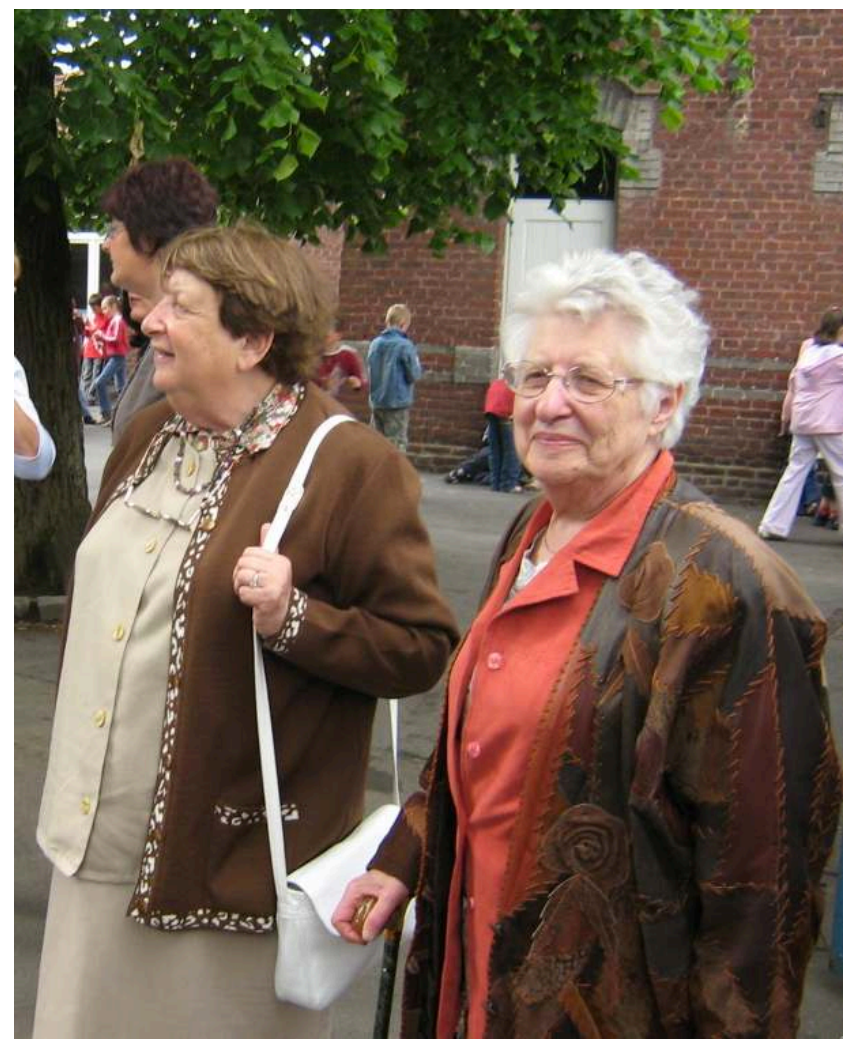

Photos de Monique Heddebaut 